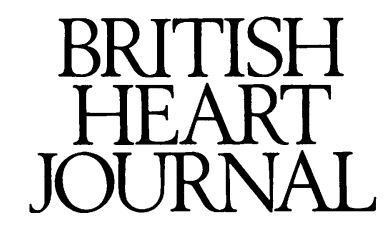

Editorial

\title{
Dynamic cardiomyoplasty for heart failure
}

In dynamic cardiomyoplasty the latissimus dorsi of the patient is lifted as a pedicle graft, placed in the chest cavity, and the muscle is wrapped around the ventricles of the failing heart. ${ }^{1}$ This muscle undergoes low frequency electrical stimulation for several weeks to confer fatigue resistance. The muscle is then stimulated by a synchronisable burst stimulator to contract during cardiac systole. ${ }^{2}$

Dynamic cardiomyoplasty has been proposed as both an alternative and a bridge to cardiac transplantation. Heart transplantation, a more established treatment, can rescue patients with terminal heart failure, often dramatically improving their quality of life. In contrast, because of high operative mortality, cardiomyoplasty is unsuitable for patients with terminal heart failure.

The improvements in the quality of life and functional class in patients in New York Heart Association class III, with some exceptions, ${ }^{3}$ are more modest than those achieved with transplantation. Nevertheless, cardiomyoplasty does not require a donor organ, there is no rejection, and no need for immunosuppression. It is also likely to cost less.

\section{Transforming skeletal muscle}

The problem of skeletal muscle becoming fatigued by repetitive contraction was overcome by a transformation technique. In dogs several weeks of low frequency electrical stimulation altered the phenotypic expression of skeletal muscle and converted a muscle made up of mixed fibres, such as the latissimus dorsi, into an almost pure type I muscle, which is highly resistant to fatigue. ${ }^{45}$ Because the muscle fibres in the myocardium are syncytial, a single electrical impulse such as that delivered by an ordinary cardiac pacemaker can elicit all-or-none contraction of the whole heart, whereas to generate sufficient power in skeletal muscle requires a burst stimulator to recruit the separate motor units. This constraint led to the development of a programmable burst stimulator that can be synchronised with cardiac systole by $R$ wave sensing. ${ }^{6}$

Mechanisms and early clinical experience

Between 1985 and May 1991 more than 100 patients worldwide underwent dynamic cardiomyoplasty. ${ }^{7}$ The surgical technique was refined, ${ }^{8}$ the transformation protocol and stimulator devices tested, and case selection criteria were examined in the light of clinical outcome. Because of high operative mortality, cardiomyoplasty proved to be unsuitable in patients who were terminally ill. The consensus that emerged was that in general only patients who were in New York Heart Association (NYHA) functional class III despite appropriate medical management should be considered for this operation. In $80 \%$ of patients who had cardiomyoplasty the functional class and quality of life improved gradually in the months after the operation. ${ }^{9}$ Improvements in haemodynamic variables, however, were relatively modest and inconsistent.

Several mechanisms could account for the improvements after cardiomyoplasty. The synchronised squeezing of the heart during systole was an effective assist mechanism in laboratory animals and in many patients. ${ }^{10}$ Cardiomyoplasty, by adding another layer of muscle to the ventricular wall, may be regarded as iatrogenic cardiac hypertrophy. In accordance with Laplace's law, the increase in wall thickness may reduce the myocardial wall tension. Experimental studies support this explanation. ${ }^{11}$ The passive presence of muscle around a failed heart may also delay progressive ventricular dilatation by modulating the remodelling process of the failing heart. ${ }^{12}$ In ischaemic cardiomyopathy wrapping stimulated skeletal muscle around the heart increased blood flow to the ischaemic myocardium..$^{13}$ The clinical trials described above had inconsistent follow up and concurrent performance of surgical procedures such as coronary bypass operation, ventricular aneurysmectomy, or valve replacement introduced confounding factors. This made it difficult to interpret the outcome data. ${ }^{8}$ Furthermore, some workers seemed biased in the selection of the medically treated patients who were compared with the cardiomyoplasty group. ${ }^{14}$

\section{Prospective studies}

Cardiomyoplasty patients were enrolled in a phase II prospective study approved by the United States Food and Drug Administration (FDA) at five centres in the United States, two in Canada, and one in Brazil between May 1991 and September 1993. Sixty patients underwent cardiomyoplasty and 58 case matched patients in the reference group were medically treated and followed. Although follow up is continuing and the results have not been published, some of the main findings were presented at the American College of Cardiology meeting in March 1994 and at the Cardiostim meeting in June 1994. As a result of earlier experience nearly all patients in NYHA functional class IV were excluded, and concomitant surgical procedures avoided. Patients (mean 
age 58 years) had a mean left ventricular ejection fraction of $22 \%: 68 \%$ had idiopathic dilated cardiomyopathy and $32 \%$ had ischaemic cardiomyopathy. There were eight procedure related deaths among the cardiomyoplasty patients and nine patients in the reference group underwent heart transplantation in the subsequent months. None the less, two years after the procedure mortality in the patients who had cardiomyoplasty was not increased. The most obvious difference between the two groups was the change in NYHA class. Twelve months after operation only $15 \%$ of the cardiomyoplasty patients remained in the same functional class, $44 \%$ had improved by one class, and $41 \%$ had improved by two classes (mean change 1.3 classes). In the reference group $7 \%$ of the patients worsened by one class and $41 \%$ remained in the same class without improvement. In $41 \%$ function had improved by one NYHA class and in $11 \%$ it had improved by two classes (mean change 0.6 class). These results were reflected in scores for quality of life: the cardiomyoplasty patients showed greater improvement, particularly in scores related to daily activities.

The cardiomyoplasty patients also showed modest but statistically significant improvement in systolic functions, including the ejection fraction, stroke volume, left ventricular stroke work index, and mean (SD) wall motion scores. Peak $\mathrm{VO}_{2}$, which was $14.4(4.7) \mathrm{ml} / \mathrm{min} / \mathrm{kg}$ before operation, remained unchanged at 12 months whereas there was some increase in the anaerobic threshold and exercise time on the modified Naughton protocol. Only a quarter of the reference patients who died had sudden cardiac death whereas three quarters of the patients who died from non-procedure related causes in the cardiomyoplasty group had sudden cardiac arrest, which in many was presumably caused by arrhythmia. Fewer patients died from pump failure. The incorporation of automatic implantable cardioverter defibrillator capabilities in the future generation of synchronisable burst stimulators may improve survival after cardiomyoplasty.

Although the number of patients who required hospital admission and the number of admissions per patient were similar in both groups, there was evidence that fewer cardiomyoplasty patients required admission to the intensive care unit, and that when they did their stay was shorter. It remains to be seen whether such savings will significantly offset the cost of cardiomyoplasty.

\section{Dichotomy between Europe and United States}

There is an interesting dichotomy in the regulatory approach adopted in Europe and the United States. In early 1994, on the basis of data obtained in the study approved by the FDA and from more than 400 patients operated upon worldwide, Medtronic was given permission to sell its stimulator in the European Union. This means that in Europe patients in heart failure can undergo cardiomyoplasty when it is deemed to be appropriate by their cardiologists and cardiac surgeons. In the United States, however, the FDA mandated a prospectively randomised control study, which may require as many as 300 patients in each arm. Thus the availability of a stimulator for cardiomyoplasty continues to be strictly limited and regulated in the United States. Since dynamic cardiomyoplasty cannot be performed without such a stimulator, the whole operative procedure, and not just the device, is restricted and cardiomyoplasty cannot be undertaken unless it is part of a prospective randomised study.

The proposed prospective randomised study that the FDA demands is clearly the most rigorous and appropriate study design, but it engenders complex logistic and ethical issues. ${ }^{15}$ A surgical procedure cannot be blinded and a placebo control is impossible. Unlike the comparison of two medical treatments, surgical and medical treatments are not equivalent in many ways. For example, cross over from one treatment to the other is always one sided-that is from medical treatment to surgery. The usual approach of analysing the results on the basis of intention to treat tends to diminish the value of surgical intervention, because a patient originally treated medically and saved by subsequent cross over to surgical treatment will be counted as a medical success. Another confounding factor in surgical trials, not evident in medical study, is the technical skill of the participating surgeons: a "learning curve" is inevitable.

The difficulties of cardiomyoplasty study are compounded by the condition of patients considered for this operation and by the stage to which the technique has developed. The first question is that of patient preference. ${ }^{16} \mathrm{~A}$ potential candidate for cardiomyoplasty will have to balance a short-term $\geqslant 10 \%$ risk of surgery against later improvement in quality of life and possibly longer survival. Only a patient who is dissatisfied with the medical treatment is likely to accept this immediate risk. Once such a patient is admitted to the study he or she may resent being randomised back to medical treatment and may refuse to participate. This could seriously slow down the recruitment of patients and, because the medical management of heart failure is evolving rapidly, a prospective control study which takes many years may become obsolete before it is completed.

Another important feature of prospective randomised studies is that the protocol mandates strict criteria, not only for case selection but also for the surgical procedure undertaken. In most previous prospective randomised studies medical treatments were compared with well established standardised surgical procedures. But the technique for cardiomyoplasty is not standardised. Is it better to use the right latissimus dorsi rather than the left, in what direction should the muscle fibres run in relation to the cardiac axis, and what is the best protocol for muscle transformation? These are among the issues that are being debated and investigated. Each surgeon uses a somewhat different technique, with outcome and the surgeon's experience and confidence guiding the selection of the best technique. A rigid definition of the surgical technique to be used in a prospective randomised study may hamper the natural evolution of the fittest surgical approach. I wonder whether heart transplantation would have developed successfully if in the 1970 s, when the mortality was still high and late survival poor, it had been allowed only as part of a prospective randomised study protocol?

What then, is the best approach? Now that cardiomyoplasty is available in Europe I hope patients will still be studied prospectively under a protocol and that reference patients will be recruited. Although such a study is not perfect, it may be the best we can get under the circumstances. Perhaps we should see how far a randomised control study can be pursued in the United States. If it can be successfully accomplished, its scientific merit will be hard to dispute: and, even if ultimately it does not prove to be feasible, the different approaches on two continents may turn out to be complementary. ${ }^{17}$

McGill University, Montreal, RAY C-J CHIU

Quebec, Canada

1 Salmons S, Jarvis JC. Cardiac assistance from skeletal muscle: a critica appraisal of the various approaches. Br Heart $f$ 1992;68:333-8.

2 Hill A, Chiu RC-J. Dynamic cardiomyoplasty for treatment of heart failure Clin Cardiol 1989;12:681-8. 
3 Chiu RC-J, Odim JNK, Burgess J, et al. Responses to dynamic cardiomyoplasty in idiopathic dilated cardiomyopathy. Am $\mathcal{F}$ Cardiol 1993; 72:475-9.

4 Salmons S, Sreter FA. Significance of impulse activity in the transformation of skeletal muscle type. Nature 1976;263:30.

5 Ianuzzo CD, Hamilton N, O'Brien PJ, Desrosiers C, Chiu RC-J. Biochemical transformation of canine skeletal muscle for use in cardiac assist devices. F Appl Physiol 1990;68:1481-5.

6 Dewar ML, Drinkwater DC, Wittnich C, Chiu RC-J. Synchronously stimulated skeletal muscle graft for myocardial repair: An experimental study. $\mathcal{F}$ Thorac Cardiovasc Surg 1984;87:325-31.

7 Carpentier A, Chachques JC. Myocardial substitution with a stimulated skeletal muscle: First successful clinical case. Lancet 1985;i:1267.

8 Chachques JC, Grandjean PA, Carpentier A. Latissimus dorsi dynamic cardiomyoplasty. Ann Thorac Surg 1989; 48:600-4.

9 Grandjean P, Austin L, Chan S, et al. Dynamic cardiomyoplasty: Clinical follow-up results. 7 Card Surg 1991;6:80-8.

10 Moreira LFP, Stolf NAG, Jatene AD. Hemodynamic benefits of cardiomyoplasty in clinical and experimental myocardial dysfunction. In:
RC-J Chiu, I Bourgeois, eds. Transformed Muscle for Cardiac Assist and Repair Mount Kisco, NY: Futura. 1990;179-88.

11 Lee KF, Dignan RJ, Parmar JM, et al. Effects of dynamic cardiomyoplasty on left ventricular performance and myocardial mechanics in dilated cardiomyopathy. F Thorac Cardiovasc Surg 1991;102:124-31.

12 Capouya ER, Gerber RS, Drinkwater DC, et al. Girdling effect of nonstimulated cardiomyoplasty on left ventricular function. Ann Thorac Surg 1993;56:867-71.

13 Mannion JD, Magno MG, Buckman PD, et al. Acute electrical stimulation increases extramyocardial collateral blood flow after a cardiomyoplasty. Ann Thorac Surg 1993;56:1351-8.

14 Moreira LFP, Seferian P, Bocchi EA, et al. Survival improvement with dynamic cardiomyoplasty in patients with dilated cardiomyopathy. Circulation 1991;84:III 296-302.

15 Giertz G. Ethics of randomized clinical trials. F Med Ethics 1980;6:55-7.

16 Kassimer JP. Incorporating patients' preferences into medical decisions. N Engl $\mathcal{F}$ Med 1994;330:1895-6.

17 Dudley HAF. The controlled clinical trial and the advance of reliable knowledge: An outsider looks in. BMF 1983;287:957-60. 